\title{
Analyse pragma-énonciative des s/citations du site d'Arrêt sur images
}

A pragma-enunciative analysis of quotations on the Web site Arrêt sur images (Freeze-frame)

\section{Alain Rabatel}

\section{(2) OpenEdition}

Journals

Édition électronique

URL : http://journals.openedition.org/aad/806

DOI : 10.4000/aad.806

ISSN : 1565-8961

Éditeur

Université de Tel-Aviv

Référence électronique

Alain Rabatel, «Analyse pragma-énonciative des s/citations du site d'Arrêt sur images », Argumentation et Analyse du Discours [En ligne], 4 | 2010, mis en ligne le 15 avril 2010, consulté le 12 octobre 2020 URL : http://journals.openedition.org/aad/806 ; DOI : https://doi.org/10.4000/aad.806

Ce document a été généré automatiquement le 12 octobre 2020

Argumentation \& analyse du discours est mis à disposition selon les termes de la licence Creative Commons Attribution - Pas d'Utilisation Commerciale - Pas de Modification 4.0 International. 


\title{
Analyse pragma-énonciative des s/ citations du site d'Arrêt sur images
}

\author{
A pragma-enunciative analysis of quotations on the Web site Arrêt sur images \\ (Freeze-frame)
}

Alain Rabatel

1 La multiplication des sites de presse sur le WEB, l'importance de leur structure hypertextuelle et des liens renvoyant vers de nouveaux discours, soulèvent de nouvelles questions en matière de dialogisme, et notamment de discours représentés (au sens générique où cette notion est entendue dans Rabatel 2008b). Dans le même mouvement, la prise en compte des effets argumentatifs produits par ces nouvelles pratiques de convocation des discours enrichit l'analyse de l'argumentation.

2 Ce qui est nouveau, c'est moins l'utilisation de l'image et du son, qui jouent un rôle de premier plan dans les médias audio-visuels, que l'intégration de ces captures d'images et de sons dans une trame écrite abondante et hétérogène à partir de laquelle les lecteurs/spectateurs sont incités à aller voir de plus près tel ou tel document, telle ou telle séquence enregistrée. Ce phénomène sera exploré sur le site d'Arrêt sur images (@si $)^{1}$ : on analysera à cette fin la représentation des dires autres dans la livraison hebdomadaire du site, nommée la Gazette, qui renvoie à des émissions phares de la semaine disponibles sur le site, ainsi que dans certaines des émissions. Ce site met en œuvre des modalités relativement originales de discours représentés, qui correspondent à des formes inédites de citations en contexte pluri-sémiotique, des $s /$ citations, selon la suggestion amicale de Laurence Rosier.

On rappellera d'abord le cadre sémiotique global du site d'@si (1), avant de dégager une première fonction argumentative du discours d'escorte, qui annonce le discours représenté/montré et le présente comme citation à faire apparaître, puisqu'il n'est lisible qu'après que le lecteur a cliqué sur lien hypertexte (2). Une deuxième fonction argumentative, plus médiacritique, considère le discours montré comme des preuves, c'est-à-dire comme des citations à faire comparaître (3). Cette exploitation argumentative des discours autres se trouve également illustrée par le procédé du 
montage, qui démonte les situations tout en servant de support à une démonstration. Le montage repose sur une logique circulaire de la justification et de la preuve; le discours montré et le commentaire du discours d'escorte s'étayent réciproquement, construisant une connivence évidente à laquelle il est difficile d'échapper, contrairement aux formes plus traditionnelles d'argumentation toujours sujettes à contestation car les arguments peuvent se voir opposer des contre-arguments à l'infini (4). Compte tenu du caractère médiacritique du site d'@si, le discours d'escorte vise certes à donner envie de cliquer sur les incrémentations et de regarder les témoignages et enquêtes, mais, surtout, il construit un ethos de professionnalisme exigeant et une communauté discursive et interprétative soudée autour de valeurs partagées. On sera ainsi amené à préciser le positionnement énonciatif de l'éditorialiste/rédacteur en chef d'@si, Daniel Schneidermann, par rapport aux énonciateurs cités (5).

\section{Analyse globale du cadre sémiotique}

4 Caractérisons globalement, d'un point de vue sémiotique, la structure de la scénographie énonciative en dégageant les caractéristiques des deux situations représentatives du site.

La Gazette se compose d'un texte d'une vingtaine de lignes environ centrées autour d'une ou deux émissions en lien avec l'actualité de la semaine, faisant l'objet de commentaires incitant le lecteur à cliquer sur le lien. Ainsi en (1), (2) et (3) :

(1) Le débat qui fait rage oppose un Alain Minc à un Bernard-Henri Lévy. Avec, en matière de propagande, un avantage à ce dernier, qui vient de publier une double page dans Le Monde, et dont on découvre qu'il n'est pas allé où il disait être allé, et qu'il n'a pas vu ce qu'il affirmait avoir vu (@si Gazette 34)

(2) La couverture du Nouvel Obs montrant Simone de Beauvoir nue traduit-elle un sexisme inconscient? Regardez le débat enflammé entre David Abiker, notre chroniqueur, et Violaine Lucas, militante de l'association Choisir, La cause des femmes. (@si Gazette 2)

(3) Pendant ce temps, sur le WEB, la base militaire fulmine contre le manque de moyens, et l'impréparation des soldats envoyés en Afghanistan. Mais on ne l'entend pas à la télévision. (@si Gazette 34) ${ }^{2}$

6 La deuxième scénographie correspond au cas où le lecteur, ayant cliqué sur un des liens, accède à la rubrique " émissions » (parmi les rubriques "dossiers et contenus », « chroniques », « vite dit » et «forum »). Toutes sont conçues sur le même modèle : un texte résume le contenu et la trame des échanges tout en renvoyant à des captures d'écran qui permettent d'accéder à la totalité de l'échange ou à ses temps forts. Ce genre de dispositif est illustré par l'exemple (4):

(4) Dan Israël, qui s'est plongé dans les reportages des guerres d'Algérie et d'Afghanistan, a repéré une première similitude: les scènes de distribution du courrier. Babey [chargé des questions de défense sur France 3] tente d'expliquer : « Dans une premier temps, lorsque vous êtes envoyé spécial, vous traitez les thèmes d'actualité ", raconte-t-il. "Mais au bout d'un moment, c'est d'un intérêt décroissant." Les angles possibles pour le reporter sont souvent les mêmes: courrier, restauration des soldats... [...]

Daniel [Schneidermann] ne résiste pas à mettre les pieds dans le plat : «Quand on interviewe des transfuges, c'est du journalisme ou de la propagande? " Regardez les débuts de réponses de nos deux invités [capture d'image] (@si, débat du 29-8-2008: «A la guerre, il y a des images propres et des images sales ») 
7 Le dispositif peut être parfois dédoublé, comme dans les cas de montage, où le texte confronte, en les surplombant, deux documents différents :

(5) Une autre spécificité de l'armée française, en Algérie comme en Afghanistan, est qu'elle tient à montrer qu'elle n'est pas seulement là pour faire la guerre. En 1959, elle s'occupait aussi de l'éducation des enfants algériens proches de ses bases. En 2008, elle forme les militaires afghans, pour qu'ils soient capables de se défendre seuls contre les Talibans.

Mais « mission pacificatrice » ou non, les soldats ne sont guère enclins à critiquer leur «mission». Et hier comme aujourd'hui, de Bigeard aux officiers postés en Afghanistan, les discours justifiant « leur » guerre ont à peine changé.

[Capture d'image]

[Capture d'image]

(@si, Gazette 35)

8 Le discours d'escorte évoque/convoque dans le texte de la Gazette des discours à venir, qui sont ensuite montrés à l'écran. Ces derniers sont représentés parce qu'ils reposent sur une situation d'énonciation distincte de celle du locuteur citant, sans subordination : la juxtaposition des espaces énonciatifs plaide donc bien en faveur de l'hypothèse du discours représenté pour les s/citations ${ }^{3}$.

Dans la Gazette, l'énonciation citante est assumée par D. Schneidermann en tant que responsable éditorial du site d'@si. L'énonciation représentée/montrée n'existe qu'en puissance d'«actualisation", car l'énoncé n'est audible/visible qu'à la condition d'ouvrir la page de l'émission. Dans les pages des émissions, l'énonciation citante n'est pas identifiable: mais il s'agit soit de D. Schneidermann, soit d'un journaliste qui assume la même fonction médiacritique et le même rôle de garant. C'est ce qu'on voit en (5), particulièrement représentatif du corpus.

Dans ces deux situations, le discours d'escorte a d'abord une visée informative. Mais il a aussi diverses fonctions argumentatives, directes ou indirectes (Amossy 2006 [2000]) car le but final est d'une part d'inciter les lecteurs à naviguer sur le site, d'autre part d'exercer une posture médiacritique et de la faire partager par les utilisateurs du site.

\section{Présenter le discours représenté/montré comme des s/citations à faire apparaître}

11 La première fonction argumentative du discours d'escorte citant introduisant au discours représenté/montré s'appuie sur les spécificités sémantiques des verbes. En effet, les verbes qui annoncent le discours cité sont rarement des verba dicendi - ou des noms qui caractérisent une interaction langagière, comme en (1), (6), (7), (8). Le plus souvent, ceux-ci sont remplacés par des verba sentiendi ou mêlés à eux, notamment à des verbes de perception visuelle ou auditive à l'instar de (6) et (7) :

(6) Notre second invité de la semaine, Jean-François Probst, ancien conseiller de Jacques Chirac et Charles Pasqua, raconte cette censure dont il a été victime. Il revient aussi sur ses liens avec Bakchich.info dont ce gaulliste est, de manière étonnante, un des fondateurs et animateurs. Pour l'écouter, c'est ici. (@si Gazette 6) (7) Cette enquête, bizarrement, est tombée dans un trou noir médiatique (un de plus). Vous devez entendre notre première invitée de la semaine, Pascale Kremer, journaliste au Monde 2. (@si Gazette 7)

De plus, les verba dicendi ou sentiendi - éventuellement accompagnés de verba movendi dans les commentaires, à l'image de (15) : «Allez regarder », « n'hésitez pas à l'envoyer 
à vos amis » - sont souvent associés à des présentatifs, accompagnés des adverbes de lieu « ici » ou « là », comme en (6), (8) et (9) :

(8) Alors que la crise se poursuit, Dumay répond à toutes ces questions avec une totale franchise. Son interview est ici. (@si Gazette 3)

(9) Toute l'histoire de la loi bafouée est là. (@si Gazette 5)

13 Ces marques ont pour fonction première d'annoncer le contenu du discours représenté /montré en le présentant de façon intéressante, comme des citations à faire apparaître: car la citation reproduit la totalité des paroles, encore plus fidèlement qu'une citation traditionnelle, puisque la capture d'images rapporte le verbatim, ainsi que le grain de la voix, les intonations, les pauses, les silences, les réticences... Bref, elle visualise la nature globale de l'interaction représentée/montrée, avec la totalité des paramètres mimo-gestuels et situationnels, avec d'éventuelles prises d'images de documents enchâssés, eux-mêmes de nature audio-visuelle. Le discours représenté/ montré équivaut encore à une citation à faire apparaître en raison du fait que le discours d'escorte souligne constamment l'intérêt qu'offrent les documents montrés, tant pour leur valeur informative intrinsèque que pour leur valeur extrinsèque, par rapport à la posture médiacritique revendiquée par Arrêt sur images. Avec cette dernière dimension, on quitte la fonction informative liée à la pluri-sémioticité des sites WEB, que nous venons de résumer sous l'expression de s/citations à faire apparaître pour entrer dans une autre dimension, plus argumentative, celle des s/citations à comparaître, voire, à faire comparaître.

\section{Transformer les s/citations à faire apparaître en s/ citations à faire comparaître}

La deuxième fonction argumentative du discours d'escorte s'appuie sur la dimension juridique métaphorique présente dans l'acte même de citer, et qu'on retrouve dans l'étymologie latine de citare :

Toute prise de voix est une authentique convocation, comme le dit le sens propre du verbe latin citare, 'appeler à comparaitre', 'convoquer', littéralement inviter à 'parler avec', à 'prendre voix avec', à se réunir en une seule voix, parler en chœur ou à plusieurs. (Ouellet $2005: 200$ )

Si l'on file la métaphore juridique, on dira que le discours représenté/montré est un discours convoqué. Il est analogue à une citation à comparaître, le tribunal étant celui de l'instance médiacritique qui instruit les affaires médiatiques en un sens éthique et politique qui n'est pas sans rappeler une certaine éthique de responsabilité (Rabatel et Koren 2008). Cette visée argumentative s'appuie sur les caractéristiques sémantiques précédentes. À la différence du prototype des verba dicendi, le verbe dire, particulièrement neutre au plan sémantique, les verba sentiendi, du fait de leur forme injonctive, présentent la caractéristique de s'adresser directement au destinataire - le lecteur d'@si. Plus, elles visent à l'impliquer, comme l'indiquent encore les verba movendi et les présentatifs, dont la dimension conative est forte dès lors que le co(n)texte incite fortement à «regarder " ou à «écouter », à l'instar de "Pour l'écouter, c'est ici », en (7). Le lien entre journalistes d'@si et destinataires est d'autant plus fort qu'il est marqué par des conseils, exprimés avec des impératifs, invitant à dépasser la surface des choses, comme en (10) ou (11). 
(10) Vous ne ressortirez pas de notre émission de cette semaine avec des certitudes. Ou, peut-être, la certitude que... dans l'incertitude, il est prudent de prendre des précautions. Notre émission est ici. Mais surtout, si le sujet vous préoccupe, ne vous en contentez pas. L'opération de David Servan-Schreiber, dont on n'a pas fini de parler, est décortiquée ici. (@asi Gazette 25)

(11) Comme la semaine dernière, notre observatoire des 'oublis' des 20 heures est fidèle au poste. Passez le lire! (@asi Gazette 30).

Le recentrement sur le destinataire est renforcé lorsque les commentaires expriment un devoir(entendre, regarder), comme en (7), ou un falloir, une nécessité à laquelle les journalistes ne peuvent pas échapper - «il nous fallait », à deux reprises, en (23). De même, le « nous » de " nous écouterons ", en (12), est sans doute à comprendre comme un nous dilaté, englobant non seulement l'équipe professionnelle d'@si, mais aussi tous ceux qui partagent sa quête de la vérité :

(12) Nous avons créé cette semaine un dossier Claude Askolovitch. Pourquoi ? Parce que ce valeureuxconfrère vient de passer du Nouvel Obs au groupe Lagardère, où il sera notamment en charge d'un éditorial politique sur Europe 1. Coïncidence? Au début de l'été, c'est lui qui avait déclenché "l'affaire Siné », en volant au secours de Jean Sarkozy, fils du président, malmené par le dessinateur de Charlie Hebdo. Il est désormais un des éditorialistes les plus influents de France. Nous écouterons attentivement cette nouvelle voix du grand orchestre du système. (@si Gazette 35)

Les exemples (13) et (14) jouent le même rôle en usant cette fois d'une adresse directe au lecteur sous la forme du " vous », ou des interactions entre " vous » et «nous » :

(13) Guiral donne une explication cohérente. Convaincante? A vous de le dire. (@si Gazette 10)

(14) Et puis, @rrêt sur images n'est pas un site fermé. A vous, ensuite, de compléter votre information en surfant sur toutes les ressources de la Toile, qui n'en manque pas. Nous vous mettons seulement sur la piste. (@asi Gazette 25)

Très fréquemment, le texte comprend des verbes invitant à un parcours de lecture informatif et vigilant comme en (10)-(14). Ces verbes visent à caractériser, tout en la construisant, une posture critique, tant chez les journalistes d'@si que chez les destinataires du site. D'où les incitations à lire/écouter de telle ou telle façon le document, à poursuivre les recherches, etc. C'est pourquoi il ne s'agit plus de citations à faire apparaître mais de citations à comparaître pour mieux exercer sa vigilance critique. Encore cette formulation ne nous satisfait-elle pas pleinement, et nous parlerions plus volontiers de citations à faire comparaittre. Par là, nous voulons signifier que l'idéal d'@si n'est pas la judiciarisation du monde (cf. les citations à comparaître du monde judiciaire), mais une société qui use de son esprit critique en confrontant les dires avec d'autres dires, avec des actions, des événements, etc. C'est cette activitéde mise en discussion et de confrontation que nous résumons sous l'expression de citation à faire comparaitre, l'idée de comparution renvoyant au tribunal de la conscience rationnelle et éthique qui cherche à prendre le plus grand nombre de garanties possibles pour délibérer honnêtement.

\section{Images, photomontages et discours des photomontages, entre démontage et démonstration}

19 La position médiacritique adoptée par le site d'Arrêt sur images est également sensible à travers le photomontage, comme dans l'exemple (15): 
(15) Tout à fait autre chose. Annonces, démentis : les couacs gouvernementaux se succèdent. 'Je n'ai jamais dit ça !' a ainsi rassuré la ministre de la Santé, Roselyne Bachelot, à l'Assemblée nationale, à propos du dé-remboursement des lunettes par la Sécurité sociale. Dans un cas comme celui-ci, tout décryptage est superflu. Place donc au montage vidéo, qui permet de juxtaposer le démenti de la ministre à l'extrait de son intervention, deux jours plus tôt, où elle proposait bien... ce qu'elle jura ensuite n'avoir jamais proposé. Allez regarder notre petit montage et, s'il vous convainc, n’hésitez pas à l'envoyer à vos amis. (@si Gazette 16) que certains discours (enregistrés eux aussi) sont faux: cette preuve repose sur l'évidence de ce qui est vu, et de ce qui est vu par tous. Le montage présente d'abord la réponse, virulente et pleine d'aplomb, de R. Bachelot à une question au gouvernement du 15 avril dans laquelle elle attaque l'opposition en profitant de la question pour répondre à " une vaine querelle » : «Je me suis demandé où les commentateurs avaient été trouver cette affaire. Je me suis fait porter le verbatim de cette émission et à aucun moment je n'ai retrouvé la trace de ce qui a été dit ». Le photomontage renvoie ensuite à 1'22" de l'émission du Grand Jury dans laquelle la même R. Bachelot répond à la question de J.-L. Séguillon ("Est-ce que certains secteurs de santé comme l'optique doivent passer complètement à la complémentaire ») en disant que «la question est posée »... Bref, l'évidence visuelle rend quasi inutile tout autre discours. Ce genre d'exemple repose sur une forme d'argumentation par le réel très spécifique. Il ne s'agit pas ici de recourir à l'exemple, au modèle (Reboul 1991 : 182) : la comparaison porte sur un dire autre confronté avec lui-même, qui est terriblement convaincant dans la mesure où le locuteur citant se fait le plus discret possible et où l'argument ad hominem est porté contre la personne par elle-même, par un autre discours et par une autre image d'elle-même, en sorte que l'effet est dévastateur pour les faces positive et négative de celui qui fait l'objet du montage.

Ce genre d'argumentation par l'exploitation pluri-sémiotique de l'image, en sus des discours, est également mis en œuvre dans les exemples (5) et, infra, (23), mais, à la différence de (15), le photomontage appelle des décryptages et, dans le même temps, il les fonde... Ainsi, en (23), le texte commence par rappeler la mémoire télévisuelle des usagers d'@si, avant de proposer un jugement («Ô surprise... ») qui est appelé à être vérifié par le recours aux images de la guerre d'Algérie et leur confrontation avec celles d'Afghanistan. Ce photomontage est ensuite complété par un questionnement auquel il est donné réponse par un entretien avec un spécialiste.

Comme le rappelle Didi-Huberman (2009: 86-87), l'objectif que Brecht et W. Benjamin assignent au photomontage est de jouer sur la distanciation afin d'éclairer le connu sous un jour nouveau, de lui restituer une « inquiétante étrangeté ». L'exploitation des photos s'explique parce qu'elles sont plus malaisément réfutables que les discours et qu'elles en disent toujours potentiellement plus, en vertu des inférences qu'elles alimentent. Ainsi, lorsque le spectateur a sous les yeux la photo d'un militaire américain dressé près du cadavre d'un soldat japonais (planche 47 d'ABC de la guerre), il voit en sus le triomphe sur le Japon, allié de Hitler :

la photo documente sans doute un moment sans l'histoire de la guerre du Pacifique, mais, une fois montée avec les autres - et avec le texte qui l'accompagne -, elle induit éventuellement une réflexion plus poussée sur les enjeux profonds de l'engagement américain dans la guerre contre l'axe Berlin-Rome-Tokyo ${ }^{4}$ (DidiHuberman 2009 : 33-34). 
ll en va de même avec le montage de (15): il démonte les mensonges de la communication de Roselyne Bachelot. Mais il va plus loin, par ses inférences, en "démontrant» (en apportant la preuve par l'image) que les politiques sont des menteurs. Cette double lecture opère aussi pour le photomontage qui confronte les reportages sur les guerres d'Algérie et d'Afghanistan (5 et 23) : derrière les similitudes de traitement de l'information pour ces deux conflits se profile l'idée que toute guerre relève d'une même manipulation. Ces généralisations sont abusives, elles relèvent des paralogismes extra dictionem, indépendants du langage. Le montage, sous son emprise "démonstrative » inductive, fonctionne comme tout argument secundum quid qui érige un argument relatif en valeur absolue (Robrieux 1994: 170-1). Certes, cette " démonstration » n'a aucune valeur scientifique, mais sa force est ailleurs, compensée par l'emprise de la doxa et des images, comme s'il y avait une solution de continuité du visible au démontré...

Il existe a minima une tension, a maxima une contradiction entre deux lignes de pente du photomontage. L'une est celle de la démonstration, l'autre celle du démontage :

Etrangeté de la distanciation : d'un côté elle montre pour susciter une démonstration ; d'un autre côté, elle montre pour produire un démontage. Brecht, d'abord, ne prétend distancier toutes choses que pour démontrer les rapports historiques et politiques où elles prennent position à un moment donné. En ce sens la distanciation est une opération de connaissance qui vise, par les moyens de l'art, une possibilité de regard critique sur l'histoire. [...] Cette connaissance advient dans une perception des différences que rend possible le montage. (Didi-Huberman 2009: 69 ; voir également 95)

Cette sur-signification, qui correspond à ce que nous avons théorisé ailleurs sous le terme de sur-énonciation (Rabatel 2004), évoque une manipulation, une forme de propagande. Mais elle est compatible avec la visée critique originelle du montage. De ce point de vue, l'arsenal rhétorico-argumentatif d'@si, avec l'inclusion du montage dans un mode de raisonnement qui privilégie la justification et la preuve, ne doit pas être rejeté au magasin des manipulations impures. Le photomontage repose sur une argumentation par la preuve, grâce à la confrontation provocante et dramatisée de deux réalités visibles rapprochées par le montage. Le fait que nous utilisions la notion de "preuve " n'est pas fortuit. Ainsi que le rappelle Marcel Conche (2002: 104), le régime de la preuve est spécifique: "S'il n'est pas possible de ne pas se rendre à une preuve, il est, au contraire, toujours possible de contourner un argument ».

Cette conception est celle qui est massivement mise en œuvre sur @si. Le document pluri-sémiotique est le plus souvent conçu comme preuve suffisante, comme preuve saturante du discours, selon une logique inférentielle (Grize 1990 : 48), et non pas selon la logique rationnelle basée sur le raisonnement syllogistique. Il s'agit en quelque sorte d'une forme de justification qui se voit à l'œil nu, qui ne passe pas par la nécessité de justifier au-delà de ce qu'@si donne à voir, tant elle va de soi.

Bien sûr, @si ne se limite pas aux montages, il offre dans ses émissions une vaste palette de controverses qui mettent en œuvre toutes les formes d'argumentation rationnelle. Mais il est intéressant de s'attarder sur la valeur de preuve qui découle de l'observation du réel montré : elle est très forte dans le montage, mais elle est toujours présente, y compris en l'absence de montage. En effet, les citations font plus que rapporter des paroles et des arguments, elles montrent l'argumenteur argumentant, et cette monstration est en elle-même une confrontation qui invite à juger des arguments, de la personne qui argumente, etc. C'est pourquoi la logique de la preuve et de la 
justification est très éloignée du modèle de l'argumentation rationnelle syllogistique, très éloigné aussi de la conception de la justification développée par M. Angenot (2008 : 441), dans la mesure où il ne s'agit pas de se justifier mais de justifier par l'image une interprétation en appui sur elle, certes, mais déjà orientée par le commentaire du discours d'escorte, comme le montre l'exemple (16) ci-dessous. Si la justification peut éventuellement faire l'économie du débat dialogique en s'en tenant à la seule ligne argumentative qui étaye sa propre thèse, force est de reconnaître que la «logique » du montage est en deçà de la justification ainsi définie (par l'économie de ses moyens), et tout aussi bien au-delà (par le caractère inépuisable de sa logique inférentielle).

Le discours représenté/montré directement est interprété à travers le prisme des actions, des comportements, et c'est cet ensemble de monstrations muettes qui argumente et se justifie, à travers cette confrontation. (16) offre un bon exemple de ce genre de pratique qui consiste à interpréter non pas les paroles, mais les difficultés à répondre, voire les silences, en se servant de tous les indices posturo-mimo-gestuels et du contexte pour étayer une thèse. Ainsi l'image d'une non réponse, ou d'une difficulté à répondre y est présentée comme « éloquente » :

(16) Quelle étrange machine, un journal de 20 heures. On le croirait parfois fabriqué par davantage de réflexes que de réflexion. Exemple cette semaine : pourquoi seul Tapie a-t-il eu les honneurs d'un passage au 20 Heures, le soir de son audition, et pas l'ancien président du Crédit Lyonnais Peyrelevade, pourtant entendu le même jour par les mêmes députés? Choix politiques des télés aux ordres ? Peut-être. Les journaux télévisés, après tout, ne se sont pas trop attardés à se demander si Sarkozy, en l'occurrence, avait donné un coup de pouce à Tapie, pour l'aider à récupérer quelques dizaines de millions.

Mais la réponse est peut-être plus rassurante et plus inquiétante à la fois. Ecoutez donc, sur le plateau, les réponses d'un témoin de choix : Jacques Asline, réalisateur du 20 Heures de TF1 depuis de longues années (et encore aujourd'hui). Inconnu du public, l'homme est présent à toutes les réunions, assiste à tous les choix. Pourquoi Tapie ? lui demandons-nous. Et pourquoi justement l'instant où il semble au bord des larmes? La difficulté d'Asline à répondre est éloquente. Je ne vous en dis pas plus. Notre émission est là. (@si Gazette 37)

Et D. Schneidermann conclut par un «je ne vous en dis pas plus » qui certes peut être interprété comme une marque de suspense... une invitation à aller voir de ses propres yeux, mais qui doit aussi être interprétée comme le signe que l'image suffit plus qu'un long discours à prouver. La nature audio-visuelle du médium renforce ce genre d'argumentation par le silence (Robrieux 1993: 176), qui permet au commentateur d'exploiter à son avantage les réactions visibles des personnes interrogées. C'est là, par rapport aux limites de la citation, la supériorité de la télévision : s'il est difficile de citer qui ne dit rien, il est en revanche toujours possible de montrer des difficultés de communication et d'interpréter (voire de sur-interpréter) le silence de celui qui est interrogé... Et c'est là, par rapport au caractère labile de la télévision, l'intérêt des sites WEB qui combinent l'image et le texte, en laissant des traces lisibles de ces enchâssements, même si les parcours de lecture sont plus ou moins aléatoires.

Toutefois, la logique inférentielle est telle qu'elle ne peut se borner à abonder dans le sens indiqué par le discours d'escorte, elle peut aussi le contester, et cela d'autant plus que le montage est loin d'être la seule façon de faire. Cela confirme la dimension critique, non manipulatrice, de ce genre de preuve. Ce type d'argumentation par le montage et, au-delà, par toutes formes de confrontations, a une fonction critique : il correspond à une des caractéristiques fortes d'Arrêt sur images, comme d'ailleurs de 
tous les autres sites qui travaillent à une critique éclairée des médias (Acrimed). Il n'est pas de véritable citoyenneté sans appel à la vigilance et à l'action critique, et c'est en quoi @si, à travers l'organisation globale de son site, vise à dépasser le simple guidage des interprétations, plaidant pour la création d'une communauté critique ouverte.

\section{Tensions entre le guidage des interprétations et la construction d'une communauté critique ouverte}

Le discours représenté/montré est proposé à partir d'un lien sur lequel le lecteur peut à sa guise cliquer ou non. Il est possible que le lecteur pressé ou peu intéressé s'en tienne à ce qui en est dit dans la page d'accueil. Cette éventualité est assez théorique, en raison de la présence des injonctions qui entourent le lien, et aussi en raison des effets de mise en valeur du témoignage, comme dans l'exemple (17) : il s'avère difficile de résister à l'injonction, à l'appel à l'exhaustivité, difficile de se dérober à un débat « nécessaire »...

(17) Ce nécessaire débat vous attend ici. Et pour aller plus loin, tous les éléments du contexte sont ici. (@si Gazette 8)

Dans la plupart des cas, le discours représenté/montré proposé vient s'insérer dans la trame d'une argumentation critique sur les médias et les pouvoirs. Toutefois, le discours de la Gazette ne se réduit pas à un guidage directif, il comprend aussi un ensemble de formulations plus ouvertes en direction des destinataires. D'ailleurs, il n'est pas sans signification que la caractérisation des mimiques et du silence, en (16), soit orientée autant vers l'objet du discours (la personnalité en question) que sur la question qui fait débat, et qui permet de construire une communauté discursive basée sur un ethos professionnel de distinction médiatique, partagée avec le lectorat, en opposition aux tenants de la médiocrité, de la désinformation, etc. Au plan pragmatique, le guidage est ainsi en tension avec l'ambition de construire cette communauté discursive exigeante d'utilisateurs et de professionnels des médias. En définitive, on retrouve ici, ce qui n'est pas étonnant, la solidarité des trois dimensions essentielles de l'argumentation aristotélicienne, une focalisation sur l'objet du discours (le logos); sur le destinataire (le pathos), en vue de trouver les arguments qui le convainquent et de s'adresser à lui dans des formes efficaces parce qu'elles répondent aux attentes que le discours construit partiellement (et satisfait) ; et, enfin, une mise en scène de la personne même de l'argumentateur, qui doit être crédible dans le rôle qu'il endosse (l'ethos).

\subsection{Ethos de professionnalisme}

Les commentaires construisent l'image d'un locuteur-professionnel qui forme à la vigilance critique des médias à travers la monstration d'un ethos de précaution, de prudence, de modestie et d'acharnement à comprendre. La page d'annonce de la gazette 25 comprend ainsi les verbes et participes "démêler ", " préoccupe, "on n'a pas fini de parler ", " décortiqué », " pisté », qui construisent la posture professionnelle du journaliste vigilant, opiniâtre et perspicace. D'une certaine façon, il s'agit de renouer avec l'image mythique du journaliste fouille-merde américain (Muhlmann 2004) qui ne s'en laisse pas conter et de se donner l'image d'un professionnel fiable s'appuyant lui-même sur un réseau d'informateurs et de sources sérieux (18), (19) : 
(18) Sans doute êtes-vous, comme nous, un peu perdus. Dans l'équipe d'Arrêt sur images, je l'avoue, il nous a fallu faire un rattrapage accéléré pour éviter de vous dire trop de bêtises sur le sujet. [...] Cette semaine, nous avons d'abord tenté de vous orienter vers les sites et les médias les plus fiables... et les plus clairs (@si Gazette 40)

Chaque discours d'escorte construit un ethos de journaliste d'investigation et d'analyste critique exhaustif, comme le montrent les adjectifs de totalité en (8) et (9), les indéfinis de quantité qui dénotent un travail important ("depuis plusieurs mois », Gazette 39) pour assurer un travail journalistique d'investigation aussi fiable et pertinent que possible. De même, les commentaires mettent en avant la nécessité de dépasser la description pour expliquer, comme en (19), (20). Et surtout, la mise en scène repose sur un positionnement d'hyperprofessionnels, par l'attitude adoptée à l'égard des sources, qui distingue @si des journalistes du champ moins exigeants ainsi que le soulignent les commentaires de (20), (21) et (22) :

(19) Comprendre. Nous servons à cela. Vous aider à comprendre. Et, pour commencer, comprendre nous-mêmes. (@si Gazette 42)

(20) Nous avons recensé les précautions que prennent les journalistes (satisfaisantes), et nous avons émis quelques hypothèses (éclairantes) sur le pourquoi et maintenant' [des révélations des manipulations des Carnets d'Yves Bertrand] (@si Gazette 43)

(21) Nous nous sommes essayés, dans l'équipe, à un exercice difficile, et plus difficile que jamais à propos d'obama: l'enthousiasme critique. Comment rester insensible à la dimension historique de l'événement, et aux images de joie des Noirs américains? Certains matins, il n'y a pas d'autre choix que de les laisser, simplement parler. Mais en même temps, il nous fallaitpointer les ridicules des présentateurs-vedettes français, fondant sur Manhattan comme des sauterelles. Il nous fallait pointer leurs a-priori parfois extravagants sur les Etats-Unis, et je vous conseille à ce sujet notre analyse des reportages de l'envoyée spéciale de France 3.

Désir de passer à présent derrière l'icône : au fond, notre invité de la semaine, le démographe iconoclaste Emmanuel Todd, est dans la même attitude. Je vous conseille sa réaction, après visionnage d'un savoureux montage de présentateurs vedettes se bousculant à Manhattan. Il y décèle un « désir de servitude ». Les EtatsUnis? «Ce n'est plus une hyperpuissance, c'est une hypernuisance ». Quant à Obama, "c'est le candidat de l'establishment financier ", assure-t-il. Vrai ? Faux ? Nous nous sommes empressés d'enquêter. (@si Gazette 45)

Ce dernier exemple manifeste une co-énonciation (Rabatel 2004, 2005) avec Todd et avec le public d'@si, pour la démarche iconoclaste, c'est-à-dire un point de vue coconstruit par plusieurs sources énonciatives qui le prennent en charge. Cette première posture se double d'une posture surplombante de sur-énonciation envers les journalistes ridicules et serviles, dans la mesure où la façon dont le locuteur/ énonciateur primaire reformule leurs comportement est uniquement à charge (à @si "l'enthousiasme critique», aux autres un suivisme "ridicule»), tout en laissant le lecteur se forger sa propre opinion, au terme d'une enquête exigeante et sans parti pris. Ces manifestations sont à lire, derrière leur forme parfois euphémisée, comme une manifestation de professionnalisme. Qui plus est, elles sont le plus souvent affichées à l'occasion d'un dialogue avec les lecteurs, comme si cela montrait la symbiose entre un public et des journalistes exigeants, qui s'entraînent réciproquement dans un cercle vertueux...

Il n'en reste pas moins que dans certains cas, cette symbiose est mise à mal, comme à propos du livre sur le Canard Enchaîné. Mais dans ce cas, l'équipe d'@si se donne le beau rôle, tout en ménageant la susceptibilité des lecteurs... 
(22) Rarement un sujet avait à ce pont enflammé nos forums. Nous avons décidé d'ouvrir un dossier sur le Canard Enchaîné à partir d'un livre à charge, publié cette semaine. Qu'avions-nous fait! Cris de colère, soupirs de déception, menaces de désabonnement: certains abonnés n'ont pas apprécié du tout. Pas touche au cher Canard!

Nous ne sommes pas canardocides: nous aimons le Canard Enchaîné. Nous nous reconnaissons dans son modèle économique sans pub. Nous nous voyons même volontiers comme un petit caneton sur la Toile. Vous voulez une confidence personnelle? Accro depuis l'âge de quinze ans, je ne pourrais pas m'en passer un seul mercredi. Mais un livre est publié. C'est une enquête sérieuse, qui pose des questions sur les influences politiques qui s'exercent sur l'hebdomadaire satirique. Au nom de quoi devrionsnous faire silence?

D'autant qu'un ancien journaliste du Canard, Nicolas Beau, reconnaît sur notre plateau qu'un scoop sur les honoraires d'avocat perçus par Sarkozy alors qu'il était ministre de l'intérieur a été enterré par l'hebdomadaire, entre les deux tours de la présidentielle, parce que Sarkozy... était une source du Canard. Si vous n'avez pas envie de le savoir, ne regardez pas notre émission de la semaine. On n'oblige personne. Personnellement, cette révélation ne m'empêchera pas de continuer à lire, et aimer le Canard. L'émission est ici. La révélation de Nicolas Beau est là. (@si Gazette 48)

Quelle est l'influence de ce genre de discours impositif? Il est certain que la marge du lecteur est réelle, et il serait intéressant d'analyser des parcours de lecture, ainsi que de pratiquer des entretiens avec les utilisateurs du site pour savoir s'ils partagent les commentaires d'@si ou si l'écoute des documents télévisuels les amène à remettre en cause ces commentaires. Mais indépendamment de ces analyses, il est important de prendre en compte la récurrence de ce genre de commentaire et les effets que cela implique en terme de construction d'une communauté discursive critique des médias et d'un ethos de professionnel des médias déontologiquement irréprochable - quelles que soient par ailleurs (voir Rabatel \& Chauvin 2006, Rabatel \& Koren 2008, Rabatel 2008a) les limites et les naïvetés de l'invocation incantatoire de la déontologie... En ce sens, ces commentaires sont plus complexes que le seul guidage de la lecture des discours représentés/montrés.

\subsection{Création d'une communauté discursive exigeante, critique et ouverte}

Cette communauté est matérialisée par des marques personnelles de dialogue entre l'émetteur et les destinataires du site, traités en allocutaire, avant de devenir éventuellement des interlocuteurs (qu'ils deviennent en participant aux forums d'@si). Parfois, les textes de présentation de la livraison sont composés d'une suite de questions, ou encore de questions réponses qui donnent lieu à de nouvelles questions, comme en (23) et (24) :

(23) Vous avez certainement vu, au journal télévisé, des reportages avec les troupes françaises d'Afghanistan. Les soldats recevant leur courrier, instruisant les sousofficiers de l'armée afghane, ou bien une interview édifiante d'un ancien taliban, rallié à l'armée officielle. 0 surprise ! Ces séquences sont les mêmes, exactement les mêmes, que celles que tournait la télévision balbutiante lors de la guerre d'Algérie, au tournant des années 60 . Regardez-les : on les dirait parfois directement inspirées l'une de l'autre.

Mystère de la communication (de la propagande ?) de guerre. Pourquoi la vie de nos soldats est-elle racontée toujours de la même manière? Est-ce l'armée qui tient la main des cameramen, en leur indiquant ce qu'il faut voir (et ne pas voir)? Question posée dans notre émission de cette semaine. Elle vous apprendra des choses: 
pourquoi de Gaulle tenait à ce qu'on voie les images d'Algérie, et comment le pouvoir d'aujourd'hui n'a pas voulu que l'on voie les cercueils des morts français, à leur descente d'avion. (@si Gazette 35) dimension polémique : par exemple les questions entre parenthèses dont la première est plutôt provocatrice, tandis que la deuxième oriente vers une authentique controverse. Mais d'autres questions échappent en apparence à cette polémicité. Ainsi l'interrogation sur les raisons pour lesquelles les militaires au combat sont toujours filmés de la même façon est une vraie question qui se situe dans une propédeutique. Il en va de même pour les questions de (16), même si leur interprétation est plus contrainte. S'interroger sur le fait de savoir « pourquoi seul Tapie a-t-il eu les honneurs d'un passage au 20 Heures, le soir de son audition, et pas l'ancien président du Crédit Lyonnais Peyrelevade, pourtant entendu le même jour par les mêmes députés? » est une vraie question, qui fait écho à l'interrogation légitime du public. La question est malgré tout restreinte en raison de l'adverbe « seul », qui est fortement en opposition au fait que Peyrelevade, un des acteurs essentiels de l'affaire, entendu par les députés, n'a pas bénéficié de ce traitement de faveur. C'est pourquoi cette première question est suivie par une deuxième qui prolonge le questionnement, tout en se présentant comme une réponse à la question précédente. Là encore, la question dialectique, en apparence ouverte sur les «Choix politiques des télés aux ordres? » - dans la mesure où elle vise la nature «politique» ou non du «choix» - est cependant contrainte par le présupposé qui suit (« des télés aux ordres »). La formulation, sans copule prédicative, entend échapper à toute contestation et se présenter comme une vérité d'évidence qui concerne l'ensemble des chaînes et des opérateurs, comme le signale l'indéfini à visée générique. La succession des questions peut se lire comme une sorte de dialogue construit à partir de questions auto-adressées et hétéro-adressées, qui miment la quête de la vérité en montrant l'honnêteté intellectuelle et l'absence de parti pris (apparent) des journalistes.

Cette dimension dialogique, dialogale ouverte est bien mise en musique par D. Schneidermann en (24) :

(24) L'équipe d'@si n'est d'ailleurs pas unanime. Regardez donc David Abiker sur notre plateau, cette semaine, s'en prendre à notre consoeur du Parisien, Odile Plichon, qui témoigne de la pression croissante exercée sur les soldats [français en Afghanistan]. Pour David, les médias pinaillent, et cherchent des poux à l'Armée. Je suis plutôt d'accord avec notre invitée dans ce débat-là, qui m'incite à revenir aux fondamentaux de la mission de la presse. Mais je suis très heureux que notre émission puisse être le lieu de ce débat. L'émission est ici. (@si Gazette 39)

41 Cet extrait construit un ethos de journalistes professionnels médiacritiques irréprochables, construisant autour d'@si un lieu de débats, dans la mesure où D. Schneidermann fait remarquer, en (24), qu'@si n'est « d'ailleurs » (comme si cela allait sans dire) "pas unanime ", concluant sur sa satisfaction qu'@si sache gérer cette diversité de points de vue : «je suis très heureux que notre émission puisse être le lieu de ce débat ». Cette remarque est d'autant plus significative que D. Schneidermann ne se positionne pas en extériorité et en surplomb par rapport à une question fondamentale : « Je suis plutôt d'accord avec notre invitée dans ce débat-là, qui m’incite à revenir aux fondamentaux de la mission de la presse ». Dans ces exemples, l'ethos des journalistes n'est pas une fin en soi, il vise à construire des valeurs partagées, celles qui permettent de définir un media citoyen. 


\section{Conclusions relatives à la posture médiacritique d'@si}

Faut-il partager la remarque de Madelon sur l'ambivalence des sites qui, comme @si, revendiquent une posture méta-critique des médias dans les médias, dans la mesure où l'éducation aux médias - plus récente que la critique des médias de masses'apparente à une «pseudo-critique » (Madelon 2008 : 114) ? En témoignerait le fait que ces discours sont prescriptifs, normatifs, qu'ils «poussent leurs destinataires à penser comme eux " (ibid. : 116). Selon Madelon, Arrêt sur images sur France 5 se caractérisait par une «imbrication étroite entre discours rapporté et métadiscours» (ibid.: 115), mêlant souvent des considérations sur éthique et déontologie sans bien définir ces notions, en sorte qu'il s'agirait de "médiacritique métadiscursive ${ }^{5}$ fortement normative. Le site d'@si correspond-il encore à ce jugement? La prudence s'impose en vertu de l'ambivalence des commentaires et du montage: ces derniers orientent, parfois selon une circularité des preuves qui peut agacer. Mais cela ne doit pas faire oublier les appels à la réflexion et la possibilité matérielle, offerte par le site lui-même, à travers ses documents et ses renvois, pour exercer un contrôle critique tous azimuts. Bref, @si ne se réduit pas aux commentaires de la Gazette, et la multiplicité des paroles représentées est riche d'ouvertures, tant la parole rapportée présente une irréductibilité indépassable, qui croît en proportion de la masse des paroles citées, qu'il est impensable de pouvoir toutes contrôler.

Mais "parler avec », ce n'est pas forcément parler en chœur ou d'une seule voix. D'où la question des postures énonciatives pour mieux entrer dans le maquis des relations interpersonnelles et de leurs valeurs pragmatico-énonciatives. Le fait que le commentaire domine les discours représentés/montrés tout en se servant de leur caution pose la question de la posture énonciative du discours citant: y a-t-il coénonciation? Ou plutôt, compte tenu du déséquilibre entre discours citant et cité, le discours citant est-il en sur-énonciation et le discours cité en sous-énonciation ? La question fait sens différemment selon qu'elle porte sur des locuteurs/énonciateurs acteurs de la société (représentants de la vie sociale, de la sphère culturelle, ou plus souvent encore, du monde politique) ou selon qu'elle porte sur les journalistes. Pour ne prendre que ce dernier exemple, fondamental dans la perspective média-critique, certaines représentations des discours cités/montrés relèvent de la co-énonciation: ainsi en (19), avec Libération, en (21), lorsque qu'@si manifeste sa proximité avec E. Todd. La posture de sous-énonciation apparaît plus rarement, lorsqu'il s'agit de rapporter des points de vue sans aller jusqu'à les reprendre totalement à son compte, comme en (22). Quant à la posture de sur-énonciation, elle est la plus fréquente, à l'instar de (21), lorsqu'il s'agit de «pointer les ridicules des présentateurs-vedettes français ». Le caractère parfois directif du discours d'escorte, s'adressant au destinataire par-dessus les énonciateurs enchâssés, confirme l'hypothèse d'une surénonciation dominante.

Nous ne tirerons pourtant pas argument du fait que la sur-énonciation domine pour conclure qu'@si est «manipulateur». En effet, l'abondance des documents, leur caractère évolutif et ouvert permet au lecteur de tracer sa voie, quitte à retourner la vigilance critique à laquelle il est convoqué contre le média lui-même. Et ce n'est pas un des moindres mérites du site que de le permettre. 


\section{BIBLIOGRAPHIE}

Amossy, Ruth. 2006 [2000]. L'argumentation dans le discours (Paris : Colin)

Angenot, Marc. 2008. Dialogues de sourds. Traité de rhétorique antilogique (Paris : Mille et une nuits)

Conche, Marcel, 2002. Confession d'un philosophe (Paris : PUF)

Didi-Huberman, Georges. 2009. Quand les images prennent position. L'œil de l'histoire I. (Paris :

Minuit)

Grize, Jean-Blaise. 1990. Logique et langage (Gap, Paris : Ophrys)

Le Grignou, Brigitte. 2008. « La télévision et ses critiques. Un journalisme en “simili” ? ", Questions de communication $14,243-262$

Madelon, Véronique. 2008. «La médiacritique métadiscursive : la pathémique comme stratégie médiatique », Semen $26,113-126$

Mandressi, Rafael (éd.). 2009. «Figures de la preuve », Communications 84

Muhlmann, Géraldine. 2004. Une histoire politique du journalisme (Paris : Le Monde/PUF)

Ouellet, Pierre. 2005. «Le fantôme de la parole. L'altérité citée », Popelard, Marie-Dominique \& Anthony Wall (éds). Citer l'autre (Paris : Presses de la Sorbonne Nouvelle), 195-210

Rabatel, Alain. 2004. « Stratégies d'effacement énonciatif et surénonciation dans Le dictionnaire philosophique de Comte-Sponville », Langages 156, 18-33

Rabatel, Alain. 2005. « Les postures énonciatives dans la co-construction dialogique des points de vue : coénonciation, surénonciation, sousénonciation », Bres, Jacques, P.-P., Haillet, S. Mellet, H. Nølke \& L. Rosier (éds). Dialogisme, polyphonie : approches linguistiques (Bruxelles : Duculot) 95-110

Rabatel, Alain. 2008a. « Pour une conception éthique des débats politiques dans les médias : répondre de, devant, pour, ou les défis de la responsabilité collective », Questions de communication $13,47-69$

Rabatel, Alain. 2008b. Homo narrans. Pour une analyse énonciative et interactionnelle du récit. Tome 1. Les points de vue et la logique de la narration. Tome 2. Dialogisme et polyphonie dans le récit (Limoges : Lambert-Lucas)

Rabatel, Alain. (à paraître). « Analyse énonciative des s/citations du site d'Arrêt sur images ", Marnette S. \& L. Rosier (éds). Discours rapportés, citations et pratiques sémiotiques (Louvain : Académia-Bruylant)

Rabatel, Alain \& Andrée Chauvin-Vileno. 2006. « La question de la responsabilité dans les médias ", Semen 22, 5-24

Rabatel, Alain \& Roselyne Koren. 2008. « La responsabilité collective dans la presse », Questions de communication $13,7-18$

Reboul, Olivier. 1991. Introduction à la rhétorique (Paris : PUF)

Robrieux, Jean-Jacques. 1993. Eléments de rhétorique et d'argumentation (Paris : Dunod)

Yanoshevki, Galia. 2009. « Les vidéoblogs dans l'élection présidentielle », Mots 89, 57-68 


\section{NOTES}

1. Le corpus comprend les 52 premières Gazettes.

2. Les fragments soulignés correspondent aux liens de la structure hypertextuelle.

3. Le lecteur trouvera une analyse plus développée de ces discours rapportés/montrés équivalents à un discours rapporté direct dans Rabatel (à paraître).

4. Le commentaire se comprend mieux si on lit le texte de Brecht accompagnant la photo : «Il a fallu que du sang rougisse une plage/ Dont nul de ces deux n'avait l'héritage./ Ils étaient contraints, dit-on, de se tuer ainsi./ Soit, soit. Mais demande encore : par qui ? (Didi-Huberman $2009: 41)$.

5. Les journalistes qui assurent la critique des médias, sur des sites tels Acrimed ou Arrêt sur images, où ils côtoient des chercheurs en sciences, en sociologie de la communication, des politistes ou des linguistes en analyse du discours médiatique, ne doivent pas être mis sur le même plan que les journalistes de la rubrique médias, qui peuvent éventuellement y faire de la critique : ces derniers occupent un espace dominé du champ, envers les journalistes et envers les critiques artistiques professionnels (Le Grignou 2008).

\section{RÉSUMÉS}

Cet article analyse différentes formes de citations dans les sites de presse sur le WEB, en prenant comme exemple le site d'Arrêt sur images. Après avoir analysé les deux grandes catégories d'enchâssements énonciatifs à l'œuvre sur le site et dégagé quelques spécificités du discours représenté/montré et du discours d'escorte qui commente le précédent, l'article montre que le discours d'escorte joue un rôle argumentatif majeur dans la mesure où il fait plus qu'inciter le lecteur à faire apparaître les citations, il les présente comme des éléments de preuve, bref, comme des citations à faire comparaître. La fonction rhétorico-argumentative de ces citations est confirmée par le procédé du montage, qui démonte les situations servant de support à une démonstration basée sur des preuves. Enfin, compte tenu du caractère médiacritique du site, le discours d'escorte construit un ethos de professionnalisme exigeant et une communauté discursive et interprétative soudée autour de valeurs partagées.

This article analyzes different forms of quotation on Web press sites, using the site Arrêt sur images as an example. After analyzing the two large categories of enunciative embeddings at work on the site and identifying the marks of direct represented/shown discourse and accompanying discourse, it shows that accompanying discourse plays a major argumentative role in so far as it does more than incite the reader to display the quotations, it presents them as elements of proof, in short, as quotations to be summoned. The rhetoric-argumentative function of quotations is confirmed through the process of editing, which dissects the situation while serving as a support to demonstration. This editing rests on a circular logic of justification based on proof. At last, considering the media critical character of the site, the accompanying discourse constructs an ethos of demanding professionalism and a united discursive and interpretative community based on shared values. 
INDEX

Keywords : accompanying discourse, direct reported/shown discourse, editing, justification, quotations to be summoned

Mots-clés : citation à faire comparaître, discours d'escorte, discours rapporté/montré direct, justification, montage

\section{AUTEUR}

ALAIN RABATEL

Université de Lyon 1-IUFM, ICAR, UMR 5191, CNRS, Université de Lyon 2, ENS-Lyon 